\title{
La emoción como clave del éxito para el desarrollo de competencias en la dirección de operaciones
}

\author{
Francisca Sempere Ripoll, Alejandro Rodríguez Villalobos \\ Recibido: 15 de Diciembre de 2018 / Aceptado: 19 de Febrero de 2019
}

\section{Resumen}

En este artículo se describen varios casos de éxito para el desarrollo de competencias en la dirección de operaciones, donde la emoción es el elemento fundamental en el proceso de enseñanza-aprendizaje. Proponemos una transformación del proceso de enseñanza-aprendizaje donde el alumno sea el protagonista de su propio aprendizaje y experimente la sinergia del conjunto de habilidades necesarias para enfrentarse a estos retos futuros. El objetivo es mejorar dicho proceso a través de las emociones (neuroaprendizaje), desarrollando actividades cuyos principales ingredientes son: el reto y el protagonismo del alumno, la experiencia compartida y el trabajo en equipo, la utilidad y el realismo del caso, la simulación y la adopción de roles, la gamificación (ludificación)..

\section{Palabras clave}

Neuroaprendizaje, innovación docente, dirección de operaciones, rol, ludificación, simulación.

\section{Justificación de la metodología y obje- tivos}

En el actual escenario empresarial, la dirección de operaciones es la responsable de sintonizar los procesos con el pulso del mercado, creando el máximo valor añadido y eliminando ineficiencias. El desafío se encuentra en cómo conseguir personas capaces de trabajar en equipo con otras personas (incluso de otras empresas), integrando su forma de pensar y coordinando su trabajo con el resto de la organización, y que logren la eficiencia de los procesos a lo largo de la cadena de valor, (Ministerio de Educación, Cultura y Deporte 2017). Es por ello, que la Universidad debe asumir el reto no sólo de formar a profesionales, sino también de dotarlos de un perfil de capacidades que va más allá de lo convencional.

Nuestro principal objetivo es facilitar al alumno una experiencia de aprendizaje lo más real posible; desarrollar un proyecto y un reto emocionante que le motive a desarrollar lo mejor de sí mismo, aprendiendo conceptos y herramientas, desplegando sus habilidades y competencias, trabajando en equipo con sus compañeros para lograr una experiencia

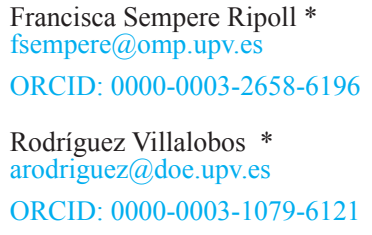

* Escuela Politécnica Superior de Alcoy Universitat Politècnica de València de aprendizaje: completa, sorprendente, emotiva, divertida, satisfactoria e inolvidable. Nuestro trabajo se basa en los últimos estudios de neuroeducación en el aula (Guillén, 2017). El propósito final es capacitar al alumno profesionalmente (a un alto nivel) para su inserción laboral inmediata en empresas exigentes y puestos de dirección con alta responsabilidad. Los objetivos cognitivos definidos a lo largo del proceso enseñanza-aprendizaje, se corresponden con los niveles de análisis, síntesis y evaluación de la taxonomía de Bloom (Bloom et al., 1956) y son los siguientes:

1. valorar la importancia estratégica y táctica de la dirección de operaciones.

2. comprender la complejidad de los procesos productivos y logísticos.

3. definir objetivos, diseñar y desarrollar planes de acción (planificar) para la gestión eficiente y flexible de los procesos productivos y logísticos atendiendo a los recursos disponibles y sus restricciones.

4. gestionar los factores clave y recursos necesarios que determinan la eficiencia de los procesos productivos y logísticos en términos de plazo, coste, y calidad del servicio a los clientes.

5. construir las funciones de producción, compras, aprovisionamiento, suministro y producción en cualquier organización a nivel de dirección (estratégico), de mandos intermedios (táctico) y a nivel operativo.

6. aplicar las herramientas y las técnicas de dirección y gestión, y en la organización de las diferentes áreas 
implicadas en el flujo productivo y logístico (compras, aprovisionamiento, producción, distribución física y logística inversa).

7. formular indicadores (KPI, Key Performance Indicators) para el análisis, la evaluación y la mejora continua (kaizen) de los procesos de forma eficiente (lean-manufacturing y lean-logistics).

8. desarrollar habilidades y competencias de: liderazgo, comunicación y presentación, coordinación, planificación, trabajo en equipo, resolución de conflictos, seguridad laboral, búsqueda de la excelencia, responsabilidad y ética profesional.

9. describir y discutir los resultados alcanzados, comparar y analizar la situación real de la empresa frente a los objetivos definidos, revisar y componer nuevos planes de acción seleccionando la mejor alternativa y justificando los cambios (proceso de mejora continua).

\section{Claves para el éxito}

Nuestra propuesta es utilizar la emoción como principal vector o motor de la motivación (Gilbert, 2005). La emoción será el principal estímulo para que el alumno pase a la acción (Tokuhama-Espinosa, 2010) y se convierta en el protagonista de su aprendizaje. Está demostrado que nuestras neuronas liberan dopamina tras experimentar emociones positivas, y que la dopamina influye en la actividad cerebral (mecanismo de recompensa y el placer) y su plasticidad. Estas experiencias emocionantes fortalecen las sinapsis y favorecen el aprendizaje. Lo que nos gusta lo aprendemos mejor (Jensen, 2008).

Para asegurar que, no sólo se active la motivación, sino que también se mantenga durante todo el proceso de enseñanza aprendizaje, nuestra propuesta es diseñada considerando los siguientes elementos clave:

1. Plantear un reto: que debe ser lo suficientemente complejo para sacar al alumno de su zona de equilibrio (homeostasis) y empujarle a actuar (motivación inicial), pero que también debe ser alcanzable (en el contexto del nivel de la asignatura) para que el alumno no se desmotive durante el proceso y alcance los resultados esperados (motivación de logro), (Hong et al., 2009).

2. Percepción de utilidad/realidad: el reto planteado debe ser establecido en un entorno real, y percibido por el alumno como algo realmente útil. Esto es esencial para la conexión del alumno desde el comienzo y debe tener la dosis de emoción necesaria para llevarle a la acción. Es importante que el alumno consiga alcanzar una visión global de los procesos y sea capaz de establecer conexiones con diferentes elementos del sistema. También debe poder determinar el impacto que una acción puede tener sobre el resto de elementos; y todo esto sólo se puede conseguir a través del diseño de modelos de simulación que se aproximen fielmente a la realidad y que permitan a los alumnos descubrir estas relaciones a través de la experimentación, (Beghetto y Kaufman, 2014).

3. Trabajo en equipo e involucración: el problema o escenario planteado debe ser lo suficientemente complejo para que no se pueda abordar de forma individual y que los alumnos tengan que trabajar en equipo colaborativamente. Se deben establecer desde el comienzo unas pautas de comunicación básicas que aseguren el buen desarrollo de la actividad, pero dejando libertad para que cada equipo establezca los elementos de comunicación informal que consideren oportunos. El trabajo en equipo fomenta la creatividad, aumenta la motivación y mantiene a los alumnos involucrados y enfocados en las tareas de aprendizaje, (Freeman et al., 2014).

4. Roles y protagonismo: la definición y asunción de diferentes roles ayuda a dar realismo a la actividad y fomentar el trabajo en equipo (sentido de pertenencia $\mathrm{y}$ aportación) desde diferentes puntos de vista (o funciones). Los roles deben ser definidos para equilibrar la participación de los miembros del equipo (Hattie, 2009) al tiempo que les proporciona protagonismo individual (pequeños momentos de éxito personal).

5. Diversión: la actividad debe incluir pequeñas dosis de diversión que permitan liberar pequeñas tensiones que se puede generar en el desarrollo de la actividad. El aspecto lúdico despierta sensaciones positivas, libera dopamina y favorece el aprendizaje (Forés y Ligioiz, 2009).

6. Sorpresa y descubrimiento: la actividad debe contener elementos inesperados y sorprendentes, lo que atraerá el interés por la actividad manteniendo el foco de atención y favoreciendo el proceso de aprendizaje (Posner et al., 2015). Las simulaciones favorecen el proceso de aprendizaje por descubrimiento mediante el proceso de prueba y error (Gruber et al., 2016).

7. Desarrollo de capacidad analítica/crítica: el análisis detallado de los resultados obtenidos es casi tan importante como el propio desarrollo de la actividad. Que el alumno sea capaz de analizar la situación con espíritu crítico forma parte del proceso de enseñanza-aprendizaje. La comparación de resultados obtenidos por los diferentes equipos a partir de una misma situación inicial refuerza y potencia el aprendizaje, ya que no sólo se aprende de lo que uno hace, sino también de lo que hacen los demás (Spaulding, 2010). 
8. Satisfacción por el logro: la satisfacción de lograr los objetivos propuestos (consecución de resultados y superación del reto) genera una emoción positiva (satisfacción y dopamina) que no sólo refuerza el aprendizaje y fija los recuerdos, sino que también alimenta la motivación del alumno y le predispone positivamente para posteriores actividades, (Jensen, 2008).

9. Percepción y reconocimiento del aprendizaje: el desarrollo de la actividad debe permitir abarcar en su totalidad el proceso de aprendizaje. Es muy importante que el alumno no sólo haya aprendido, sino que también sea capaz de reconocer y valorar el grado de aprendizaje alcanzado a través de los conocimientos adquiridos. Invitar a la reflexión del alumno en este sentido es importante para cerrar el proceso de enseñanza aprendizaje, (Tokuhama-Espinosa, 2014).

Es difícil cuantificar la medida o el peso que deben de tener los anteriores elementos. La importancia relativa de los mismos variará según el tipo de materia, los alumnos, objetivos del proyecto, etc. Pero según nuestra experiencia, todos ellos deberían tenerse en consideración de uno u otro modo para lograr una experiencia de éxito.

\section{Casos de éxito}

En este artículo se presentan dos casos de éxito, uno enfocado a la organización del trabajo y la gestión eficiente de la producción (Puertas S.A.), y otro relativo a la gestión y mejora del proceso logístico de preparación de pedidos y expediciones (LLOG). Ambos casos se corresponden con proyectos de docencia inversa realizados por los profesores autores de este artículo y que han sido premiados en la Universitat Politècnica de València. En estos, se combinan diferentes metodologías: el aprendizaje basado en el juego (GBL, Game-Based Learning), el aprendizaje basado en proyectos
(PBL, Project-Based Learning), el aprendizaje basado en la experiencia (Learning-by-doing), los juegos de rol (RP, Role-Playing), la simulación de procesos (Simulation) y la ludificación del aprendizaje (Gamification of learning). Ambas experiencias fueron creadas originalmente para el Máster Universitario en Ingeniería de Organización y Logística (MUIOL), si bien actualmente también están siendo utilizadas en el Grado de Administración y Dirección de Empresas.

\section{Puertas S.A.}

Puertas S.A. es un juego de simulación desarrollado en la asignatura de Organización de Trabajo (tamaño medio de grupo 25 alumnos) del Máster Universitario de Ingeniería de Organización y Logística (MUIOL) que simula el funcionamiento de una planta de fabricación de puertas, sobre el que se han diseñado diversas actividades de aplicación de conceptos relacionados con el área de organización de empresas y el lean-manufacturing.

El objetivo principal es que el alumno se enfrente a un sistema de producción real y comprenda el funcionamiento y la problemática asociada a su gestión pudiendo aplicar los conceptos aprendidos para su mejora.

El juego parte de un sistema de fabricación previamente diseñado en el que los alumnos pasan a ser los principales protagonistas del juego adoptando distintos roles para cada una de las secciones de la línea de fabricación: dirección de producción, planificación, almacén, diseño y corte de puertas, montaje de puertas, acabado de puertas, control de calidad, expedición y cliente. Uno de los elementos clave es el carácter físico-manual del mismo (Figura 1); la experimentación adquiere un papel muy importante porque permite al alumno entender la complejidad del sistema, al facilitar la visualización del flujo real tanto físico como de información entre los distintos puestos (identificar el origen real de los datos). 
Figura 1 Imágenes del trabajo realizado en algunos de los puestos de trabajo. Learning by doing.
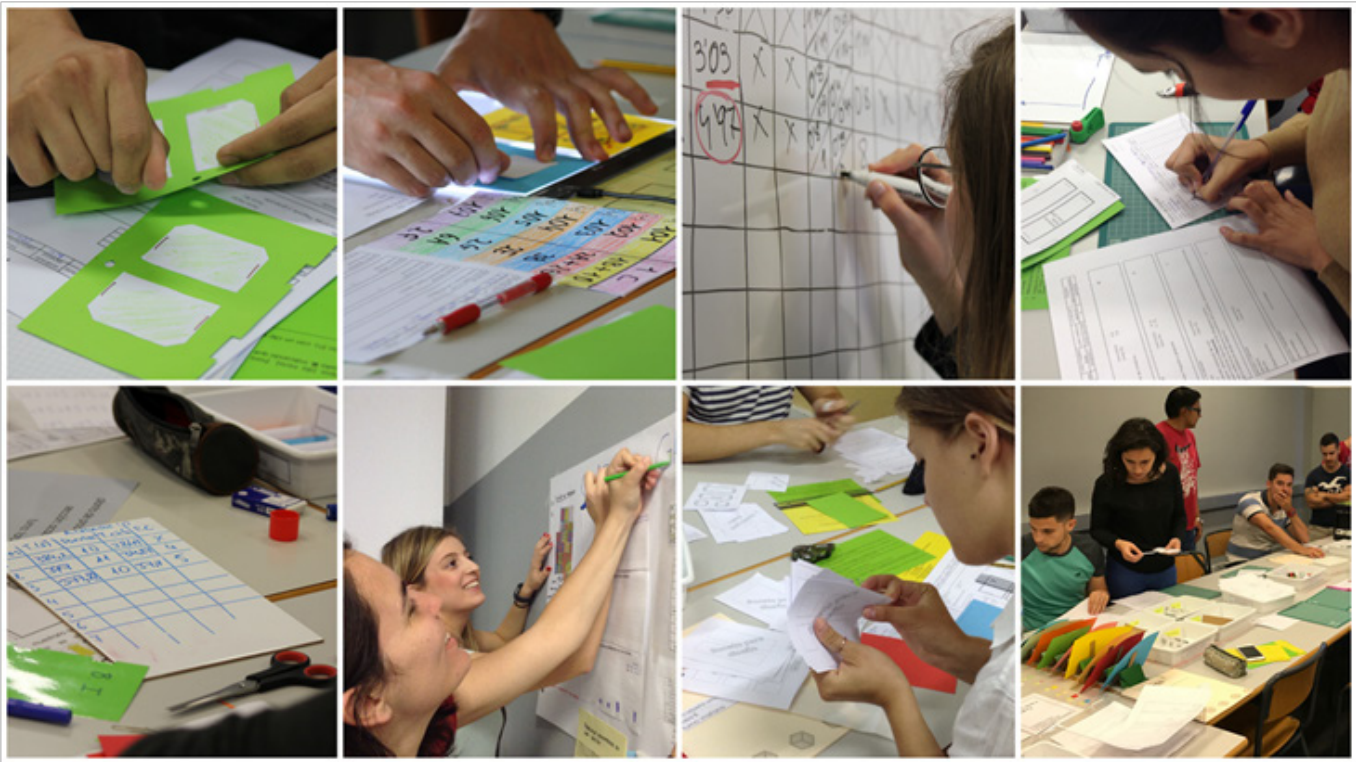

El juego ha sido cuidadosamente diseñado para albergar numerosos aspectos de la problemática real de un sistema de fabricación: órdenes de pedido, órdenes de fabricación, muestras de diseño, criterios de calidad, partes de trabajo, partes de calidad, descripción de puestos, asignación de responsabilidades, muestrarios de calidad, partes de pedido, albaranes de salida y etiquetas de expedición, entre un sin fin de documentos que soportan la dinámica de la práctica.

Actualmente el juego se lleva a cabo en las últimas 5 sesiones de la asignatura, de duración entre 4 y 5 horas cada una.
Las sesiones 2, 3 y 4 se realizan en sesiones consecutivas (mañana, tarde y mañana). El resto de sesiones, la primera y la última, tienen una semana de separación.

El punto de partida es la simulación de la planta de fabricación en la que se pone en marcha la fabricación real de puertas utilizando papel en vez de madera. El juego parte con un sistema de trabajo previamente definido, cada uno de los puestos está diseñado y dispone de una descripción detallada de las tareas y herramientas y material de trabajo a través de unas cartulinas verdes y amarillas respectivamente (ver figura 2)
Figura 2 Lay-out del puesto de almacén al inicio de la primera sesión

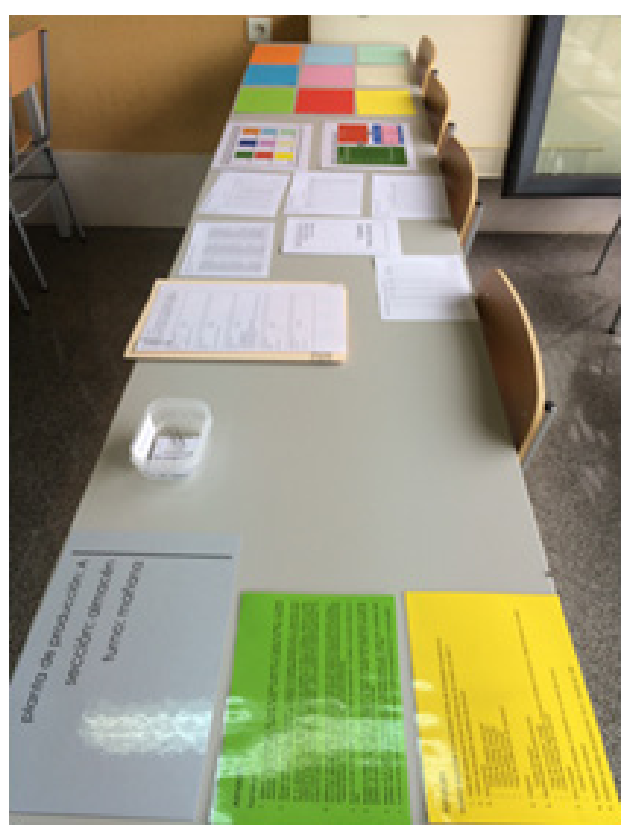


La sesión inicial tiene una duración de 5 horas, y abarca una fase previa a la simulación en la que el equipo debe entender el funcionamiento de la línea, la simulación en si misma y el análisis de los resultados. El alumno se enfrenta a los problemas derivados de la operativa general y del sistema de comunicación formal e informal que se lleva a cabo. La experimentación ayuda al alumno a identificar las relaciones entre los distintos roles y lo conciencia del impacto que su trabajo tiene en el funcionamiento del sistema. Que el alum- no actúe como fuente generadora de datos, no solo facilita su análisis posterior (por el conocimiento que adquieren en la generación de los mismos) sino que además se enfrenta a la problemática real asociada a la captura de datos.

Cada línea de fabricación se asigna a un equipo de trabajo que podrá estar formado entre 11 y 18 personas, siendo el tamaño ideal 13 personas con la distribución que se muestra en la figura 3.

Figura 3 Lay-out inicial propuesto en la primera sesión de simulación

Puertas Sh

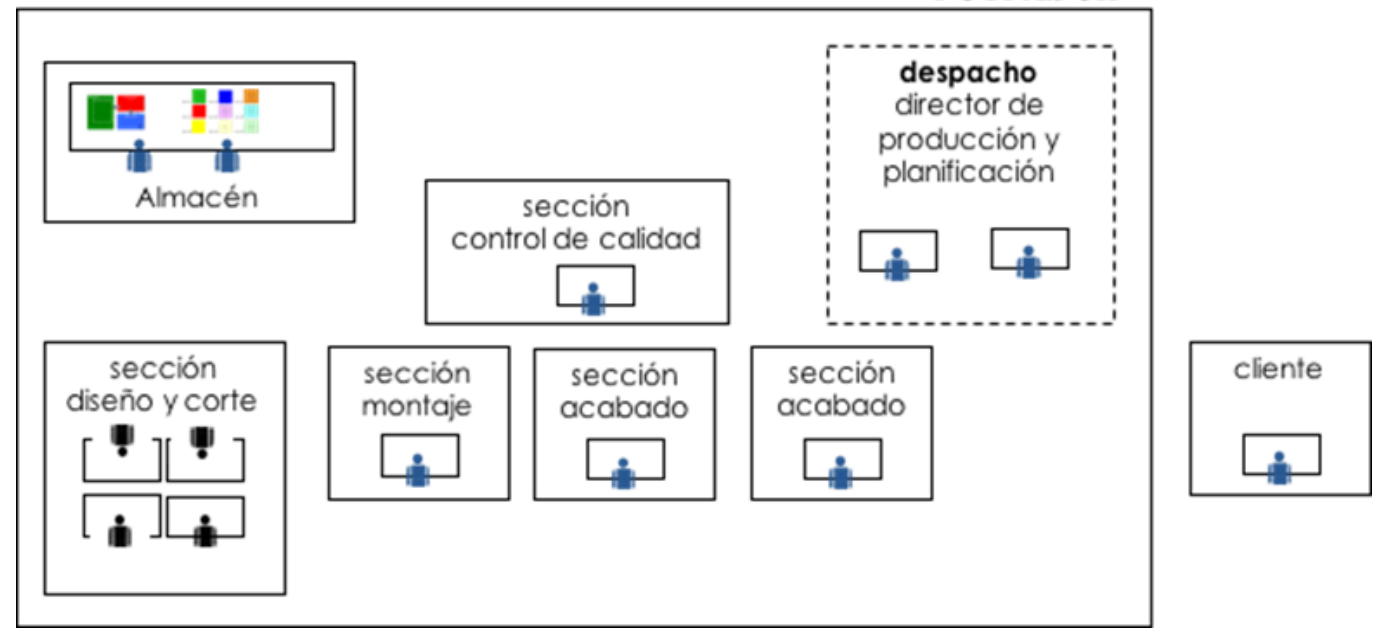

El puesto "cliente", es un puesto externo a la empresa. Cuando en una misma sesión participan distintos equipos, este puesto lo debe ocupar un participante de otro equipo. Con esto aseguramos que las puertas realmente cumplen con la calidad exigida. La experiencia demuestra que salen muchas puertas que no cumplen las especificaciones de calidad del cliente y han sido aceptadas por la sección de control de calidad.

Se recomienda realizar la selección del director de producción, antes de la sesión de simulación, con el objetivo de que el director de producción adquiera un conocimiento previo del funcionamiento de cada uno de los puestos. Hay que tener en cuenta que una vez empezada la simulación todas las dudas y problemas que se presenten en la planta deberán ser solucionadas por el director de producción (el profesor no debe intervenir en ningún caso).

Deben ser los propios alumnos los que se presenten voluntarios para ocupar el puesto de director de planta ya que se necesitan alumnos que asuman el reto de dirigir a un equipo y que estén realmente dispuestos a dedicar un poco más de tiempo que el resto de componentes del equipo. La experiencia demuestra que generalmente son los alumnos con actitud más positiva hacia el aprendizaje los que se presenta voluntarios para este puesto. El sistema de puntos de Puertas SA, otorga al director de producción 20 puntos extra por su labor.
El director de producción de cada planta deberá estudiar en detalle toda la información relativa a cada puesto y visualizar los videos explicativos de cada puesto antes de la simulación general (en el caso de que se dispongan de ellos). Es conveniente realizar una tutoría con el director o directores de producción para dar respuesta a las posibles dudas que y comprobar que conocen bien el funcionamiento de la planta.

El resto de componentes del equipo deberán consultar la información general del funcionamiento de la planta (memoria y video explicativo en el caso de disponer de él) también antes de la sesión de simulación de la planta. En el caso que no fuera posible realizar este trabajo previo, la sesión de simulación debe tener una parte inicial de duración aproximada de 1 hora, en la que se explique a todo el equipo el funcionamiento de cada puesto.

Suponiendo que tanto el director de producción cómo el resto de componentes del equipo han realizado sus tareas previas, la sesión de simulación de planta empieza con la asignación de personas a puestos. Es el director de producción quien elige a su equipo y decide en que puestos ubicar a cada componente del equipo. En el mejor de los casos los alumnos ya se conocen y les es fácil realizar esta selección. La duración estimada es entre 5 y 10 minutos.

El profesor realizará una explicación general del funcionamiento de la planta y de las reglas de la simulación (10 minutos aproximadamente) 
Cuando cada componente del equipo esté en su puesto de trabajo, dispondrá de entre 15-20 minutos para montar el puesto de trabajo y familiarizarse con el mismo. Si el puesto no ha sido montado previamente, el alumno deberá organizarse el puesto siguiendo las instrucciones que se indican en la cartulina verde ("al iniciar la sesión") y tomado como ejemplo la imagen del lay-out del puesto)

El alumno debe conocer en detalle las funciones del puesto (deben leer la cartulina verde "funciones del puesto") y revisar si dispone de todo el material necesario (comprobar cartulina amarilla "material disponible"). Si se dispone de más información del puesto (videos explicativos u otro material), es conveniente revisarla.

Los puestos más complejos, en cuanto a desarrollo de tareas, son el de Planificación y el de Almacén, por lo que, tanto el director de producción como el profesor, dedicarán tiempo adicional a la resolución de dudas de estos puestos. En el caso de que la simulación se estuviera realizando con varios equipos al mismo tiempo, se recomienda reunir a los responsables de las distantes plantas para atender conjuntamente las dudas.

Se abrirá un pequeño turno de resolución de dudas generales de cada puesto que serán atendidas por el director de producción bajo la supervisión del profesor (duración aproximada: 10 minutos)

La simulación real no debe empezar mientras haya dudas en el funcionamiento de la misma. Se requieren, por lo tanto, entre 40 y 50 minutos para la explicación del funcionamiento y resolución de dudas. Este tiempo se podría reducir si cada participante estudiara en detalle su puesto de trabajo antes de acudir a la simulación de la planta.

La simulación empieza cuando el reloj se pone en marcha. En este momento, el director de producción es el único responsable de lo que ocurra en la planta, y deberá ser capaz de dar respuesta a todas las dudas y problemas que se presentan durante la simulación. En esta primera simulación, el reloj no para hasta que el periodo de simulación programado haya finalizado.

Una vez transcurrido el tiempo preestablecido, los operarios deberán dejar sus tareas, aunque dejen el trabajo a medias, y pasar a realizar las tareas que se indican en la cartulina amarilla. Esta cartulina recoge las instrucciones a seguir para que todos los datos generados queden ordenados en una misma carpeta general dentro de sus carpetas correspondientes.

La duración estimada de una sesión de simulación dependerá de los días que se quieren simular (7-10 días), por lo que estaría de entre 1 hora y hora y media. No hay que dejar tiempo para que el equipo empiece a solucionar los problemas, aunque esa sea la tendencia habitual, es necesario que en las sesiones posteriores se analicen los problemas con técnicas de causa-efecto y no se comentan los errores típicos de las empresas de no identificar el origen del problema y solucionar los problemas "apagando fuegos". Una vez terminada la simulación, el equipo de trabajo dispondrá de 2 horas para analizar la situación inicial y definir los parámetros de productividad que ayudarán a gestionar la línea de producción.

Si el objetivo de aprendizaje de la simulación fuera: "Conocer el funcionamiento del sistema de recogida de datos de una planta de fabricación", todos los participantes deberán ocupar todos los puestos por lo que durante la simulación los alumnos irán rotando de puesto. En este caso se recomienda que cada alumno permanezca al menos 2 días en cada puesto. La duración aproximada de una sesión de este tipo es de 4-5 horas. Para reducir el tiempo de simulación a 2 horas, el tiempo de cada participante en cada puesto se deberá reducir a 1 día de simulación (8 minutos).

El equipo dispondrá de 2 sesiones y media más, de 4 a 5 horas por sesión para analizar las causas de los problemas detectados y diseñar un plan de mejora que evite el problema desde su causas raíz, además deberá rediseñar y mejorar la eficiencia de la planta a través de la aplicación de las herramientas de lean-manufacturing: VSM (Value Stream Mapping), 5'S', estandarización (trabajo y tiempo), paneles de control, cuadro de mando, paneles de marcha, puesta en marcha de talleres de mejora, equilibrado de líneas (tack time), diseño de puestos, flujo pull, diseño de un plan de polivalencia/policompetencia, puesta en marcha de un sistema de ideas de mejora, etc. Adicionalmente, el equipo podrá utilizar y aplicar conceptos y herramientas estudiadas en otras asignaturas. Durante estas sesiones se irán realizando simulaciones de 8 minutos (equivalentes a un día de trabajo real) para ir probando las mejoras implementadas, con pausas diarias donde los equipos realizan reuniones de GAPs para analizar el funcionamiento diario de la línea, solucionar problemas no previstos y establecer nuevos planes de mejora. Los equipos se enfrentan por lo tanto a la operativa real de una línea de fabricación, con problemas derivados no solo de la asignación y realización de tareas, sino también de planificación, coordinación, comunicación o recursos humanos, entre otras. En la medida de lo posible, se intentará que durante estas sesiones cada equipo trabaje en un espacio de trabajo distinto, para que cada equipo desarrolle sus propias ideas y no se vea influenciado por el resto de equipos. Antes de finalizar la cuarta sesión, se realiza una última simulación real de la planta ya mejorada, donde cada equipo recoge información del funcionamiento de la misma, a partir de la cual desarrollará un informe detallado que expondrá en la siguiente sesión al resto de equipos.

En la última sesión, cada equipo expone el nuevo proyecto de planta de fabricación y el sistema diseñado para su gestión al resto de equipos. La comparativa de resultados obtenidos entre distintos equipos de trabajo forma una parte muy importante del proceso de aprendizaje, poniendo en evidencia que no solo los aspectos técnicos son importantes en el funcionamiento de un sistema, sino que los aspectos de 
relaciones humanas tales como la formación, habilidad, comunicación, actitud, conocimientos, liderazgo o motivación juegan un papel, si cabe, más importante todavía.

Cabe destacar la enorme implicación y entrega de los alumnos en el juego, así como la complicidad que se crea entre los componentes del equipo.

\section{LLOG}

El segundo caso de éxito que presentamos es LLOG. Basado en un almacén en miniatura (Figura 4), el objetivo de LLOG es el de intentar trasladar al aula una experiencia logística lo más real y profesional posible.
Figura 4 Algunas imágenes del juego de simulación LLOG con los alumnos del MUIOL (Máster Universitario en Ingeniería de Organización y Logística)

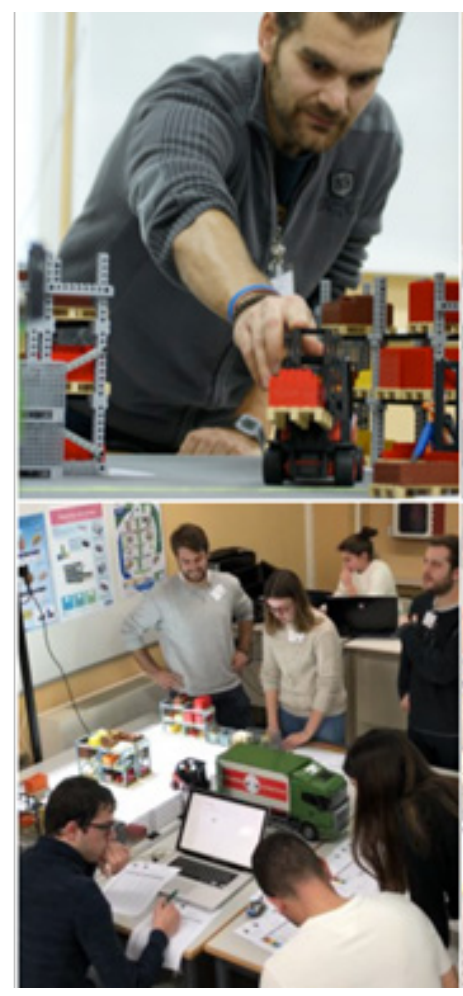

Se puede considerar que es un juego de rol y de simulación logística. Se trata de experimentar en primera persona todas las acciones y decisiones que tienen lugar en el proceso de preparación de pedidos y de expediciones desde un almacén (gestión de inventarios, decisiones de ubicación, estiba y desestiba, rutas de picking, reposición de productos, gestión de la información, consolidación de pedidos, cálculo de rutas de reparto, eficiencia, trabajo en equipo, liderazgo, etc.).

LLOG está diseñado para enseñar con emoción. Durante las sesiones, los alumnos experimentan diferentes emociones positivas (asombro, curiosidad, motivación, interés, empatía, concentración, entusiasmo, comprensión, confianza, alegría, logro, satisfacción, felicidad). La experiencia LLOG tiene tres tipos de materiales y facetas que se interrelacionan:

1. lo físico: la superficie de juego y todos sus elementos se deben tocar. En LLOG hay que mover físicamente los productos y los palets, los vehículos y los operarios. Las cosas ocupan un volumen que es importante aprovechar de forma eficiente. Se puede ver en tres dimensiones, es real y está a escala. Se aprende haciendo y tocando cosas. Todos los elementos del juego (estanterías, vehículos, personas, palets, mercancía)

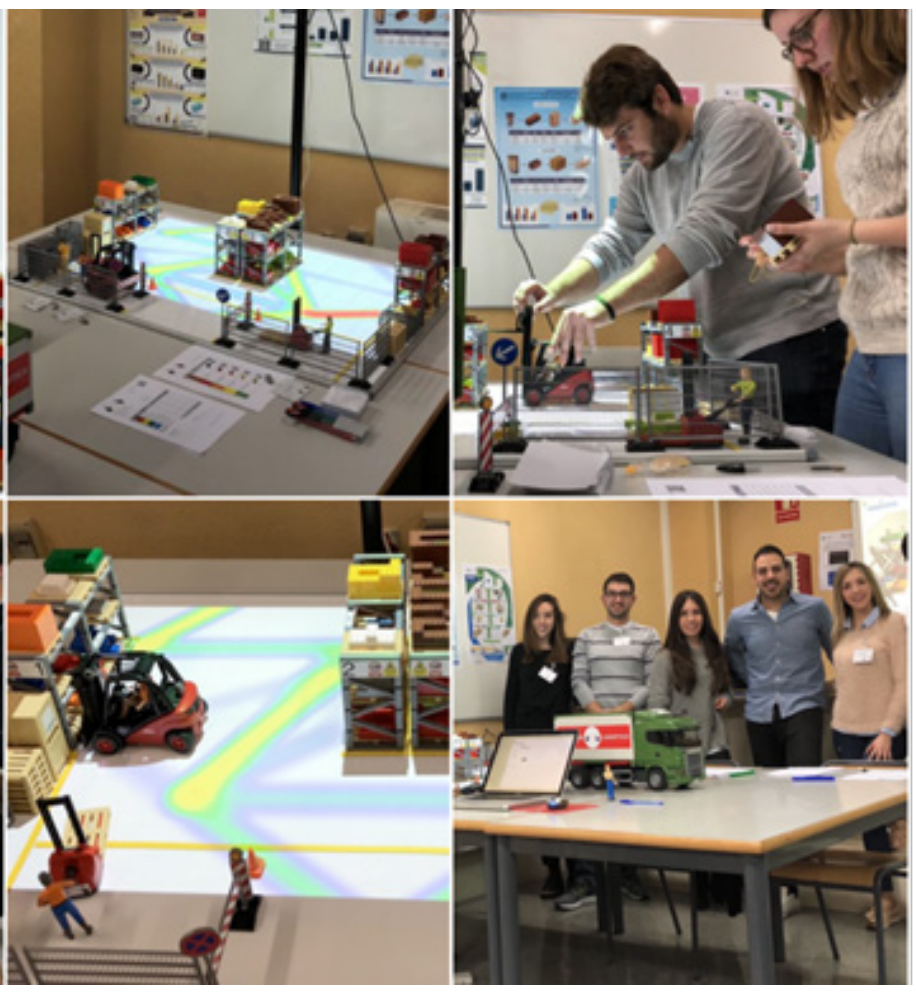

están hechos a escala. La dinámica y el sistema de información también reproducen lo que ocurre en las empresas. Esto permite simular diferentes escenarios, aprender de los errores y la experiencia, y dar el paso al mundo profesional fácilmente. Las estanterías fueron diseñadas e impresas en 3D para adaptarse a los requerimientos del juego.

2. la lógica: el profesor ha programado un software SGA (Sistema de Gestión de Almacén, o en inglés WMS Warehouse Management System). Con el software, los alumnos aprenderán la importancia de la gestión de la información ligada a los clientes, los pedidos, las ubicaciones, los inventarios, etc. Los alumnos podrán gestionar los inventarios con un verdadero lector de códigos de barras (aprendiendo de paso, su utilidad, los flujos de información y la estructura de la base de datos), y se enfrentan al reto de la trazabilidad, y de la coherencia entre el sistema lógico (el sistema de información) y el sistema físico (la realidad). El software de LLOG registra y analiza en tiempo real toda la actividad del almacén. También calcula y representa los indicadores logísticos (inventario, inventario medio, saturación, rotación, cobertura, Pareto, recorridos, rutas) necesarios para la gestión y la mejora continua del proceso. 
Incorpora un sistema de representación del almacén en tiempo real (2D y 3D) así como un sistema innovador de proyección de información e indicadores en tiempo real sobre el tablero.

3. la humana: las personas y su inteligencia. En LLOG se juega formando un gran equipo, como si de una mini empresa se tratara. Cada alumno asumirá un rol: director/a de operaciones, jefe de almacén, preparador de pedidos (picker), reponedor, consolidación y expediciones. Cada uno tendrá asignado un papel, unas funciones y responsabilidades, cada uno tendrá algo que aportar y hacer. Pero todos tendrán que colaborar e interactuar eficientemente. La información y los productos pasarán de unos a otros y tendrán que afrontar y resolver problemas colaborativamente. Son las personas las que marcan la diferencia, por eso en LLOG son el pilar de la experiencia. Los alumnos tienen la oportunidad de cambiar a un papel diferente del que tienes en la empresa, y comprender el proceso desde otro punto de vista. La planificación, la coordinación e integración, y la comunicación entre las personas son vitales para tener éxito.

LLOG forma parte de la asignatura Logística del Máster Universitario en Ingeniería de Organización y Logística (MUIOL). El tamaño medio de grupo en el máster es de unos 25 alumnos. La actividad se organiza formando equipos de entre 7 y 9 alumnos. Como se ha comentado, en cada equipo se reparten una serie de roles (con funciones, actividades y responsabilidades asignadas). El equipo es liderado por un Director/a de Operaciones y un Jefe/a de Almacén.

Los alumnos pueden elegir libremente en qué equipo integrarse, normalmente lo eligen por preferencia de horario en las sesiones; salvo que el profesor intenta repartir los alumnos extranjeros en diferentes equipos para facilitar su integración. A los alumnos españoles también les beneficia aprender a trabajar con personas de otras nacionalidades, lengua y forma de pensar. Esta actividad está calendarizada en el último tercio de la asignatura: Unidad didáctica 3 - Logística de Distribución.

Brevemente se describen las sesiones en las que se divide todo el proyecto de aprendizaje indicando las actividades, duración y el lugar donde tienen lugar:

1. Lección 'El proceso de preparación de pedidos y expediciones' (3 horas): se pueden utilizar video-apuntes (clases grabadas) de cursos anteriores, diapositivas y apuntes. El alumno debe visualizar la lección antes de la experiencia práctica, lo hace en su domicilio. El profesor atiende sus dudas y preguntas mediante tutorías a la carta.

2. Casos de estudio y lecturas (6 horas): como complemento a la lección anterior, el alumno debe realizar una serie de lecturas y casos de estudio. Lo puede hacer en la biblioteca o en su domicilio. El profesor atiende sus dudas y preguntas mediante tutorías a la carta o en el seminario. Las lecturas, los casos de estudio y la lección son imprescindibles para poder abordar con éxito la siguiente actividad práctica.

3. Sesión activa 1 (3 horas): Se forman equipos y se les presenta el reto y todos los materiales. Los alumnos se reparten los roles y comienzan a trabajar activamente en: la comprensión y planteamiento del problema, la valoración del reto y la planificación de las actividades y trabajo del proyecto. Todo ello se realiza en el aula de forma presencial. El profesor los acompaña durante la actividad, les hace preguntas motivadoras, les plantea diferentes puntos de vista, les advierte de posibles errores, etc. al tiempo que les da libertad para que aborden el proyecto según ellos mismos. El profesor observa todas sus acciones y decisiones y toma nota para su posterior evaluación.

4. Sesión activa 2 (3 horas): En esta segunda sesión presencial en el aula, y tras la anterior, el equipo realiza la simulación completamente. Registrando todos los resultados e indicadores necesarios. Nuevamente el profesor los acompaña y guía, observando y analizando todo el trabajo para su evaluación. Al final de esta sesión se hace una pequeña puesta en común y se les presenta el tipo de informe y presentación que deberán realizar.

5. Análisis de los resultados y desarrollo de un informe (8 horas): Los alumnos, trabajando en equipo deben escribir un informe para su posterior presentación acompañado de un póster resumen. En el informe describen y analizan toda la actividad realizada, su planificación, sus decisiones y acciones, los resultados e indicadores obtenidos, y unas conclusiones finales. El informe lo escriben coordinadamente entre todos, cada alumno aporta más detalle sobre el rol o función que desarrolló en la simulación. Este trabajo lo realizan los alumnos en sus casas, biblioteca o aula de libre acceso. El profesor atiende sus dudas durante el desarrollo.

6. Presentación del informe y del póster (3 horas): En una última sesión común y presencial para todos los alumnos de todos los equipos, estos hacen una exposición de sus trabajos y posters. Los alumnos comparten sus experiencias y aprendizaje, responden preguntas del profesor y de otros compañeros de otros equipos. Los informes son entregados previamente al profesor en el apartado de Tareas de PoliformaT (intranet de la UPV), y los posters son expuestos en el aula para que sean vistos por todo el mundo.

7. Evaluación (16 - 24 horas): El profesor realiza la evaluación de todos los alumnos a partir de todas sus anotaciones, trabajos, posters y preguntas. La evaluación de esta Unidad Didáctica contribuye en gran medida 
$(25 \%)$ a la evaluación continua de la asignatura. Se divide en dos partes. En primer lugar, la evaluación de la actividad mediante docencia inversa:

- $\quad$ Observación de la actividad (peso 40\%) evaluación de participación activa y competencias desarrolladas

- $\quad$ Informe redactado (peso 30\%) - evaluación del trabajo redactado

- $\quad$ Presentación de los resultados y poster (peso 20\%) - evaluación de comunicación, síntesis, etc.

- $\quad$ Preguntas respondidas (peso 10\%) - evaluación de análisis y conocimientos

Seguidamente, la evaluación de conocimientos en el control: en el segundo examen de la asignatura aparecerán 2 o 3 preguntas relacionadas con todo lo anterior (peso $20 \%$ sobre la nota del control).

\section{Resultados}

Para medir el impacto en la satisfacción del alumnado y en el proceso de aprendizaje, se utilizan indicadores cuantitativos y cualitativos. El año 2012 se toma como año de control o referencia, ya que el presente proyecto de docencia inversa comenzó en el curso 2013/14 y prosigue en la actualidad.

Los indicadores cuantitativos son los que exige el sistema educativo: las calificaciones obtenidas por los alumnos y las encuestas de profesorado. Si bien el proceso de evaluación de los alumnos se ha adaptado a las nuevas metodologías $\mathrm{y}$ al uso de la docencia inversa, este no ha cambiado en lo sustancial, y siguen existiendo diferentes pruebas de evaluación: entrega y presentación de trabajos en equipo, pruebas objetivas y subjetivas, observación y evaluación continua del desempeño de los alumnos. Además, la complejidad y el nivel de exigencia ha ido creciendo paulatinamente con los años. Como se observa en la Figura 5, las calificaciones obtenidas por los alumnos son cada vez mejores; la nota media ha pasado de 7.55 (referencia de control) a un promedio de 8.28 en los últimos 5 cursos.

Figura 5 Evolución de la calificación de los alumnos.

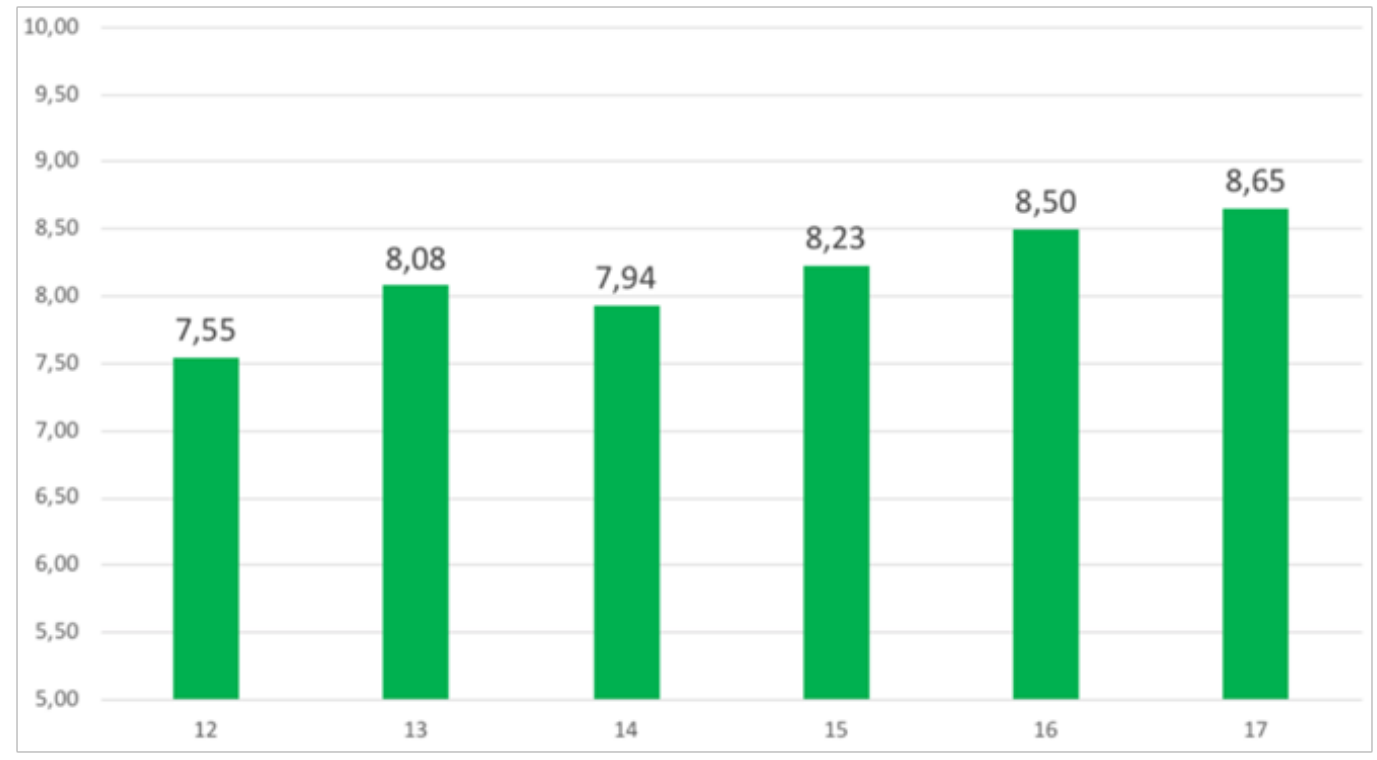

En los últimos 5 años, las encuestas de evaluación del profesorado involucrado en este proyecto de docencia inversa han mostrado una mejora sustancial. Alcanzando una valo- ración próxima al 9.5 en una escala de 10 , sobre una nota de referencia inicial próxima al 8.5 (Figura 6). 
Figura 6 Evolución de la evaluación del profesorado (azul) respecto de la evaluación media del resto de profesores del departamento (rojo).

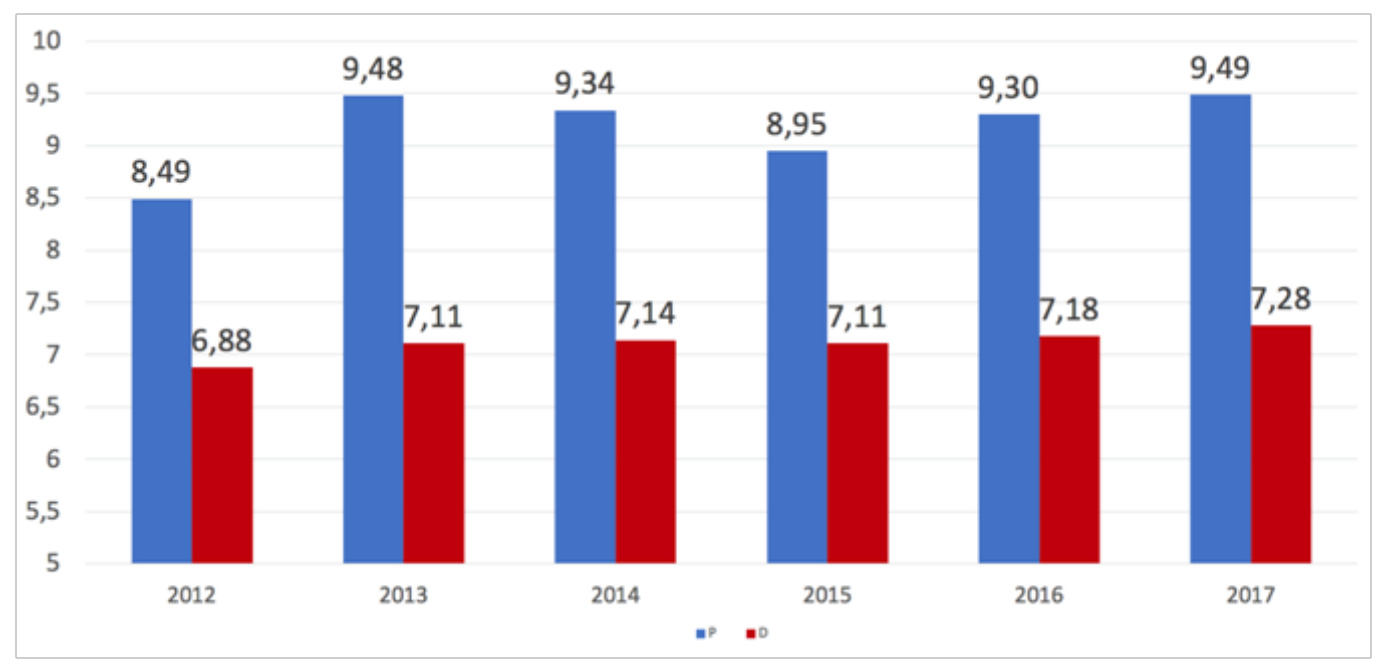

dos en estos proyectos de innovación docente es superior en todos los aspectos evaluados a la evaluación media de los profesores de su departamento. Se puede observar que los ítems mejor valorados por los alumnos son: conocimiento de la materia, motivación/interacción/ayuda y satisfacción general con el profesor. También se observa que con los años se ha ido mejorando en la evaluación de la organización y planificación, así como en la del desarrollo de la metodología docente de estos proyectos. Esto ha propiciado una menor dispersión de la evaluación del conjunto de ítems.
Figura 7 Evolución de cada ítem de la evaluación del profesorado (abajo) respecto de la evaluación media del resto de profesores del departamento (arriba).

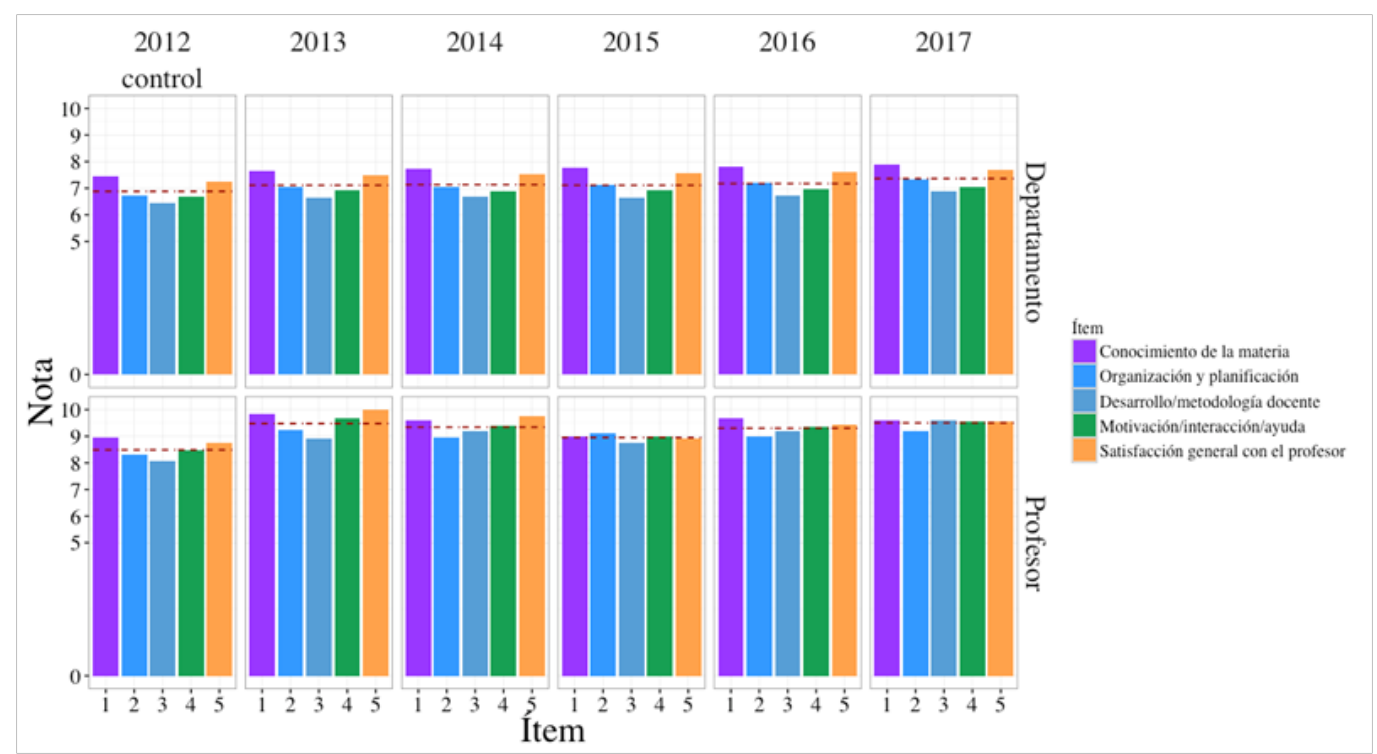


Para evaluar los aspectos cualitativos, se realizan encuestas con preguntas abiertas. En las que el alumno tiene la oportunidad no sólo de destacar los aspectos que más le han gustado, sino también de señalar los puntos débiles y aportar las mejoras que considere oportunas. En este aspecto las valoraciones han sido todas muy positivas. Lo que refleja claramente la satisfacción del alumnado y la consecución de los objetivos de aprendizaje propuestos. Entre sus opiniones, los alumnos demandan incrementar la complejidad de la experiencia. El reto está pensado para que sea asequible y motivador, pero los alumnos a medida que lo van superando demandan mayor complejidad y realismo si cabe. Los aspectos que resultan más atractivos para los alumnos son: el realismo de la simulación, el trabajo en equipo, los roles, la aplicación de conceptos, la visión global del sistema, la conexión con otros conceptos no relacionados con la dirección de operaciones, seguido de elementos tecnológicos.

Hay que destacar que tanto Puertas S.A. como LLOG han sido dos proyectos de innovación docente premiados. Puertas S.A. recibió el primer premio de Docencia Inversa Universitat Politècnica de València, concedido por el Vicerrectorado de Recursos Digitales y Documentación (curso 2016/17). En el curso siguiente, LLOG también recibió el primer premio de Docencia Inversa Universitat Politècnica de València, concedido por el Vicerrectorado de Recursos Digitales y Documentación (curso 2017/18). Ambos premios suponen un reconocimiento por parte de la Universitat Politècnica de València a la innovación docente y la mejora del proceso de enseñanza-aprendizaje, que se enmarca en una línea de acción de intensificación del uso de las nuevas tecnologías de la información y las comunicaciones en la docencia como elemento facilitador de la metodología docente a aplicar en el aula y en la implantación de innovaciones metodológicas en los procesos formativos. En el curso académico 2017/18 han sido 421 profesores impartiendo 351 asignaturas en todos los centros UPV los que han participado en el Proyecto Docencia Inversa UPV.

\section{Conclusiones}

A través de diferentes indicadores cuantitativos y cualitativos, poniendo como ejemplo dos casos de éxito reales en la docencia universitaria (en la materia de dirección de operaciones), y según nuestra experiencia; podemos concluir que la emoción debe ser un elemento fundamental en el proceso de enseñanza-aprendizaje. El proceso de enseñanza-aprendizaje debe evolucionar, haciendo protagonista al alumno de su propio aprendizaje y preparándole para enfrentarse a los retos futuros. En este artículo hemos subrayado un conjunto de elementos clave que deben ser ingredientes imprescindibles de ese proceso de transformación (el reto, la utilidad, el trabajo en equipo, el protagonismo del alumno, la diversión, la sorpresa y el descubrimiento, el análisis crítico, la satisfacción por el logro, y reconocimiento del propio aprendizaje).
Los alumnos están deseosos de participar en retos realistas, que le aproximen a la realidad empresarial. Deben sentir que están aprendiendo herramientas y conceptos útiles para su futuro laboral. Quedan atrás otros modelos más tradicionales de enseñanza como las clases magistrales o el uso de metodologías no activas. Los alumnos/as son los mejores maestros. Hay que escucharlos, observarlos y tener empatía para poder comprender sus necesidades y poder diseñar las mejores herramientas y estrategias de aprendizaje. Son fuentes inagotables de creatividad, cuando están motivados y se implican en el proceso demandarán y exigirán del profesor mucho más. Si el profesor responde, comienza un círculo virtuoso de mejora continua que es muy satisfactorio y exitoso en sus resultados y que una vez iniciado no tiene fin.

\section{Referencias}

Beghetto R. A. y Kaufman J. C. (2014). Classroom context for creativity. High Ability Studies, 25, 53-69.

Bloom, B. S., Englehart, M. D., Furst, E. J., Hill, W. H., y Krathwohl, D. R. (1956). Taxonomy of educational objectives. Handbook 1: Cognitive domain. Longmans, Green. New York.

Forés, A. y Ligioiz, M. (2009). Descubrir la neurodidáctica: aprender desde, en y para la vida. UOC. Barcelona.

Freeman S., Eddy S. L., McDonough M., Smith M. K., Okoroafor N., Jordt H. y Wenderoth M. P. (2014). Active learning increases student performance in science, engineering, and mathematics. PNAS, 111(23), 8410-8415.

Gilbert, I. (2005). Motivar para aprender en el aula. Las siete claves de la motivación escolar. Paidós, Madrid.

Guillén, J. (2017). Neuroeducación en el aula: de la teoría a la práctica. CreateSpace Independent Publishing Platform, Madrid.

Gruber M. J., Ritchey M., Wang S. F., Doss M. K., Ranganath C. (2016). Post-learning hippocampal dynamics promote preferential retention of rewarding events. Neuron, 89 (5), 110-1120.

Hattie, J. (2009). Visible learning. A synthesis of over 800 meta-analyses relating to achievement. Routledge, New York.

Hong J. C., Ming-Yueh Hwang, Chin-Hsieh Lu, Ching-Ling Cheng, Yu-Chen Lee y Chan-Li Lin (2009). Playfulness-based design in educational games: a perspective on an evolutionary contest game. Interactive Learning Environments, 17(1), 15-35. 
Jensen, E. (2008). Brain-based learning: the new paradigm of teaching. Corwin, London.

Ministerio de Educación, Cultura y Deporte (2017). Panorama de la educación indicadores de la OCDE 2017. Informe español. Secretaría General Técnica, Madrid.

Posner M. I., Rothbart M. K., y Tang Y. Y. (2015). Enhancing attention through training. Current Opinion in Behavioral Sciences, 4, 1-5.

Spaulding, L. S., Mostert, M. P. y Beam, A. P. (2010). Is Brain Gym ${ }^{\circledR}$ an effective educational intervention? Exceptionality, 18, 18-30.
Tokuhama-Espinosa, T. (2010). The new science of teaching and learning: using the best of mind, brain, and education science in the classroom. Columbia University Teachers College Press, New York.

Tokuhama-Espinosa, T. (2014). Making classrooms better: 50 practical applications of mind, brain and education science. Norton, New York. 